\title{
Application of shortening time test in battery electric range calculation of PEV based on CLTC- $\mathbf{P}$
}

\author{
Xiaopan An*, Yu Liu, Hanzhengnan Yu, Zhichao Liu, Songbo Qi, and Yang Wang \\ China Automotive Technology and Research Center Co., Ltd, China
}

Keywords: battery electric range, shortened time test, CLTC-P.

\begin{abstract}
In order to obtain the actual results of battery electric range test, and meet the demand of fiscal subsidy policy introduction of new energy vehicles, some modifications of the standard GB/T 18386.1 Test Method for Energy Consumption and Range of Electric Vehicles- part1: Light-duty Vehicles are revised by drafting group. Such as China Lightduty vehicle Test Cycle for passenger car (CLTC-P), Shortened time test method, etc. To prove the reasonability of Shortened time test to measure battery electric range (BER) based on CLTC-P, two sets of vehicle tests were carried out. Results show that shortened time test greatly reduce test time, and the weighting factor setting of DS1 and DS2 is reasonable. Although some deviation between Shortened time test and consecutive cycle test exist, but not regularly. It is related to the vehicle energy regulation strategy individually. Finally, it is recommended that the test vehicle should be forced to break 10 minutes after the end of $\mathrm{CSS}_{\mathrm{M}}$.
\end{abstract}

\section{Introduction}

With the release of finance and taxation policies of new energy vehicles, relevant technical standards shoulder the responsibility of strictly controlling technical indicators. According to the policy Notice on Further Improving the Fiscal Subsidy Policy for The Promotion and Application of New Energy Vehicles issued on March 26, 2019, subsidies for new energy vehicles will decline by 50 percent in the second half of 2019. In this notice, the battery electric range (BER) requirement is even more stringent. The threshold of subsidies has been raised to $250 \mathrm{~km}$, and those above $400 \mathrm{~km}$ have also been cut by 50 percent compared with the previous year. Besides, the reference of GB/T 36980 Energy Consumption Limits for Electric Vehicles in the policy also means that the energy consumption index would face more and more severe challenges [1].

The market has replaced the policy as the real driver of consumption, and the performance advantage of products has become an important focus [2]. As the fittest are eliminated and the strong survive, the technical department bears the responsibility of making the judgment rules. In order to improve the screening of energy conservation and emission reduction

\footnotetext{
* Corresponding author: anxiaopan@catarc.ac.cn
} 
vehicles, the technical departments has carried out the revision of relevant standards. Such as energy consumption field, more than 10 standards related to pure electric vehicles, hybrid electric vehicles and fuel cell vehicles are being revised.

In practice, however, in addition to reducing price subsides, the "range anxiety" caused by the fact that actual BER fails to reach the announcement value will also affect their choice [3]. Xu Hao's research shows that actual road energy consumption of pure electric vehicles (PEV) is $7 \%-10 \%$ higher than that of NEDC type approval value [4]. In An's study, the difference is $5 \sim 29 \%$ [5]. This is also a major pain in the NEV industry.

After more than a year of research and discussion by the drafting group, the GB/T 18386.1 Test Method for Energy Consumption and Range of Electric Vehicles- part1: Light-duty Vehicles were released in 2020. This standard has been cited by the fifth-stage (Stage V) fuel consumption regulations and the parallel corporate average fuel consumption (CAFC) and new energy vehicle (NEV) credit schemes in future 2021 [6]. Relative to GB/T 18386-2017, the revision has many modifications, such as test mass, test cycle, procedure, calculations and so on. Two core points should be paid attention to. One is the China Light-duty vehicle Test Cycle (CLTC), the first self-developed independently in China, is applied for the first time. Another is Shortened time test should be chosen for vehicles whose BER is above the prescribed value [7][12]. The Shortened time test was originally proposed by GTR15 and used to energy consumption test in accordance with the Worldwide Harmonized Light Vehicle Test Procedure (WLTP) [8]. It is improved in GB/T 18386.1-2020, such as changing the target speed curve from WLTC to CLTC, setting the number of test cycle, setting the speed value of constant segment, etc.

Therefore, in this paper, the critical revision points of the Shortened time method based on CLTC, including the test procedure and calculation process, will be firstly illustrated with text and chart. Then, so as to study whether the stipulated method can accurately reflect the actual BER, two different vehicles are selected for the Shortened time test and consecutive cycle test respectively.

\section{2 enervgy consumption test experiments}

\section{1 tesvt cycle}

The Standardization Administration of China released GB/T 38146 China Automotive Test Cycle (CATC) in 2019, which is the first independently developed driving cycles reflected actual road characteristics. Simultaneously, GB/T 18386.1 is the first national standard to quote this CATC.

According to GB/T 38146.1, the China Light-duty vehicle Test Cycle for Passenger car (CLTC-P) is applicable to M1 vehicle. Compared with the NEDC, the CLTC-P is transient operating conditions, which has a long duration and a long cycle length [9]. The version $2^{\text {nd }}$ of EV-TEST, released in 2019, has also taken the CLTC-P as the test cycle, more than twenty-eight vehicles have been tested so far [10]. 


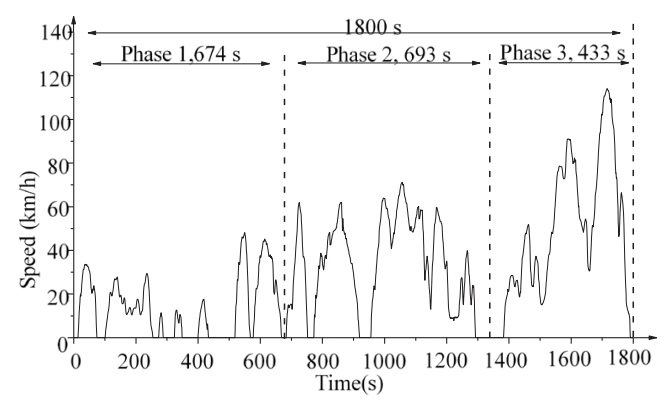

Fig. 1. Target speed of CLTC-P.

\subsection{Battery electric range test}

According to electric range test procedure, the test vehicle should be fully charged first, then a method for energy consumption test is selected to carry out the test, and finally the battery is recharged again to measure the electric obtained from the main. There are two methods for energy consumption test, one is consecutive cycle test and another is Shortened time test. Measure REESS current and voltage during the test. The current is negative when discharging. After the test, charging and measuring electric energy $\mathrm{E}_{\mathrm{AC}}$ for REESS.

Two methods of test and calculation are described in detail below.

\subsubsection{Consecutive cycle test}

The consecutive cycle test is to run the vehicle continuously in accordance with the applicable test cycle until the break-off criterion has been reached. This method is also the PEV energy consumption test method has been used. The operation mode of this method is similar to the power consumption behavior of daily travel. Therefore, it is considered to reflect the actual energy consumption accurately.

\subsubsection{Shortened time test}

The Shortened time test, originated from WLTP, is designed to complete the experiment in a short time. When the standard drafting group of GB/T 18386 introduced this method, it took into account the different test cycles and modified it according to local conditions.

The driving trace consists of follow four parts: dynamic segment 1(DS1), constant speed segment $\mathrm{CSS}_{\mathrm{M}}$, dynamic segment 2(DS2) and constant speed segment $\mathrm{CSS}_{\mathrm{E}}$. Each dynamic segment DS1 and DS2 consists of two CLTC-P test cycle. These two segments $\mathrm{CSS}_{\mathrm{M}}$ and $\mathrm{CSS}_{\mathrm{E}}$ are discharging at high speed, just to shorten the test time.

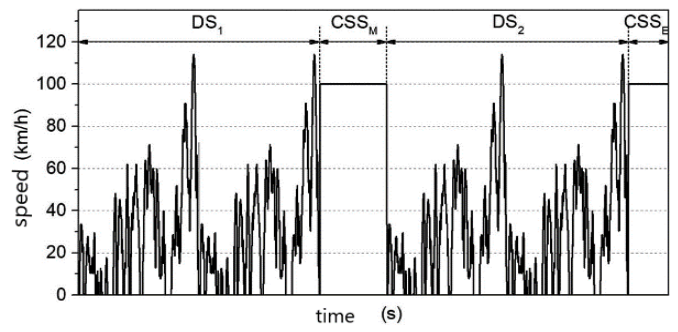

Fig. 2. Target vehicle speed trace of Shortened time test. 


\subsection{Calculation of bER}

In GB/T 18386-2017, the BER is shown directly on the chassis dynamometer equipment. In GB/T 18386.1-2020, the BER is calculated based on the electric energy change of REESS. A series of new calculation formulas are defined. The calculation process is briefly summarized as follows.

Firstly, the test data collected by the chassis dynamometer and electric quantity measuring equipment are performed preprocessing. Divide the data so that the data of each CLTC-P belongs to a separate data set. Named dataset $\mathrm{c}^{\text {th }}$ in time sequence. The parameters in dataset $\mathrm{c}^{\text {th }}$ are vehicle speed, REESS current, voltage, electric energy. For each dataset, calculate its distance $\left(d_{c}\right)$ and energy change $\left(\Delta E_{R E E S S, \mathrm{c}}\right)$.

Then, calculate the weighted electric energy consumption $\left(E C_{D C}\right)$ using the equation 2. The $K_{c}$ in the formula is weighting factor of dataset $c^{\text {th }}$. It is obtained by combining $E_{R E E S S, C C P}$ and $\triangle E_{R E E S S, \mathrm{c}}$, where $E_{R E E S S, C C P}$ is the usable REESS energy determined from the beginning until the break-off criterion reached. The $E C_{D C, c}$ in the formula is the electric energy consumption of dataset $\mathrm{c}^{\text {th }}$ according to equation 4 .

Finally, calculate battery electric range (BER) using the Equation3.

The electric consumption (EC) based on the BER and the recharged electric energy from the mains $\mathrm{E}_{\mathrm{AC}}$ shall be calculated using Eq.5. BER is a reflection of REESS output ability, whereas the EC is a reflection of utilization capacity of the external energy. This study only focuses on the BER and does not discuss the EC.

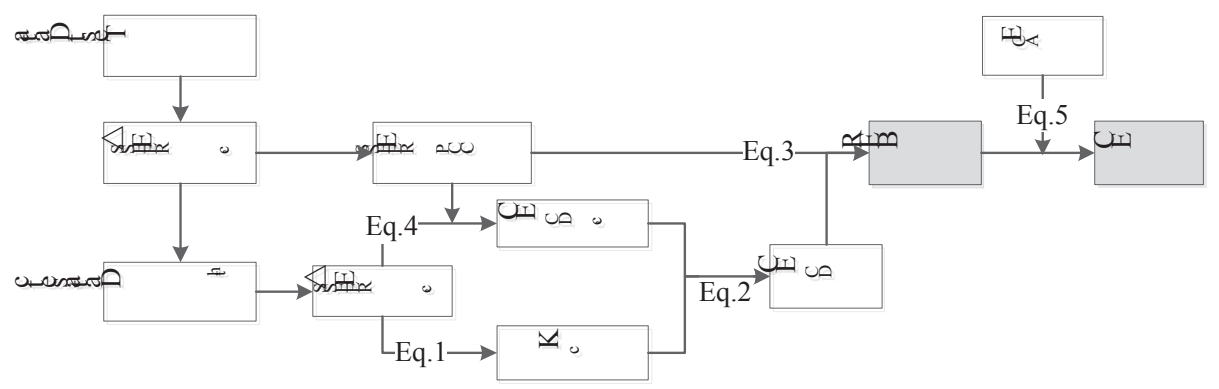

Fig. 3. Calculation flow chart of BER.

$K_{c}=\frac{\Delta E_{R E E S S, \mathrm{c}}}{E_{R E E S S, C C P}}$ for $c=1,2$ and

$K_{c}=\frac{1-K_{1}-K_{2}}{n-2}$ for $c>2$ in consecutive cycle test

$K_{c}=\frac{1-K_{1}-K_{2}}{2}$ for $c=3,4$ in shortened time test

$$
\begin{aligned}
E C_{D C} & =\sum_{c=1}^{n}\left(E C_{D C, c} \times K_{C}\right) \\
\mathrm{BER} & =\frac{E_{R E E S S, C C P}}{E C_{D C}} \\
E C_{D C, C} & =\frac{E_{R E E S S, c}}{d_{c}} \\
\mathrm{EC} & =\frac{E C_{A C}}{\mathrm{BER}}
\end{aligned}
$$

There are some difference between consecutive test and shortened time test in calculation. The shortened time test, only four CLTC-P data of DS1 and DS2 are extracted respectively for calculation. The data of CSSM and CSSE are not involved in calculation. The setting of the weighting factor in Shortened time method is determined by considering that except for the special energy consumption performance of the first two cycles, the energy consumption values of the other cycles are similar. 


\section{Results discussion}

\subsection{Results}

\subsubsection{Test vehicle A}

Test vehicle A is a domestic passenger car. The consecutive cycle test lasted for about 17 hours (excluding preconditioning). When running to the 31 st cycle, the break-off criterion has been reached. The results calculated in line with chapter 2.3. The final calculation results of consecutive cycle test are shown in Table 1a) (some intermediate data is omitted here).

The shorten time test lasted for about 5 hours. By the end of the test, the length $\mathrm{d}_{\text {CSSM }}$ of CSSM is $248.6 \mathrm{~km}$ and the length $\mathrm{d}_{\text {CSSE }}$ of CSSE is $29.8 \mathrm{~km}$. The final calculation results of shortened time test are shown in Table1b).

Compare Table 1a) with Table 1b), which indicates the difference of BER obtained by calculation in two different test methods. And it's about $2.3 \%$ shorter than in consecutive cycle test.

\subsubsection{Test vehicle $B$}

Test vehicle B is an import passenger car. The consecutive cycle test lasted for about 17 hours. The shorten time test lasted for about 6 hours. The final calculation results are shown in Table2.

Table 1. a) vehicle A-The final calculation results of consecutive cycle test.

\begin{tabular}{|c|c|c|c|c|c|c|c|}
\hline & $E_{R E E S S, C C P}$ & $d_{c}$ & $\Delta E_{R E E S S, \mathrm{c}}$ & $E C_{D C, c}$ & $K_{c}$ & $E C_{D C}$ & BER \\
\hline & $\mathrm{Wh}$ & $\mathrm{km}$ & $\mathrm{Wh}$ & $\mathrm{Wh} / \mathrm{km}$ & & $\mathrm{Wh} / \mathrm{km}$ & $\mathrm{km}$ \\
\hline 1 & \multirow{5}{*}{56440} & 14.579 & 2341 & 160.570 & 0.041 & \multirow{5}{*}{127.162} & \multirow{5}{*}{443.843} \\
\hline 2 & & 14.603 & 1913 & 131.003 & 0.034 & & \\
\hline 3 & & 14.579 & 1876 & 128.673 & 0.033 & & \\
\hline & & & & & & & \\
\hline 30 & & 14.642 & 1840 & 125.659 & 0.033 & & \\
\hline
\end{tabular}

Note: The intermediate steps in the calculations are not be rounded.

Table 1. b) vehicle A-The final calculation results of shortened time test.

\begin{tabular}{|c|c|c|c|c|c|c|c|}
\hline & $E_{R E E S S, C C P}$ & $d_{c}$ & $\Delta E_{R E E S S, \mathrm{c}}$ & $E C_{D C, c}$ & $K_{c}$ & $E C_{D C}$ & BER \\
\hline & $\mathrm{Wh}$ & $\mathrm{km}$ & $\mathrm{Wh}$ & $\mathrm{Wh} / \mathrm{km}$ & & $\mathrm{Wh} / \mathrm{km}$ & $\mathrm{km}$ \\
\hline 1 & \multirow{4}{*}{54271} & 14.519 & 2365 & 162.887 & 0.044 & \multirow{4}{*}{125.16} & \multirow{4}{*}{433.60} \\
\hline 2 & & 14.536 & 2121 & 145.909 & 0.039 & & \\
\hline 3 & & 14.508 & 1759 & 121.239 & 0.459 & & \\
\hline 4 & & 14.539 & 1799 & 123.734 & 0.459 & & \\
\hline
\end{tabular}

Table 2. a) vehicle B-The final calculation results of consecutive cycle test.

\begin{tabular}{|c|c|c|c|c|c|c|c|}
\hline \multirow[t]{2}{*}{$\mathrm{c}$} & $E_{R E E S S, C C P}$ & $d_{c}$ & $\Delta E_{R E E S S, \mathrm{c}}$ & $E C_{D C, c}$ & $K_{c}$ & $E C_{D C}$ & BER \\
\hline & Wh & $\mathrm{km}$ & Wh & $\mathrm{Wh} / \mathrm{km}$ & & $\mathrm{Wh} / \mathrm{km}$ & $\mathrm{km}$ \\
\hline 1 & \multirow{5}{*}{81525.158} & 14.461 & 2854.97 & 197.420 & 0.035 & \multirow{5}{*}{182.10} & \multirow{5}{*}{447.691} \\
\hline 2 & & 14.508 & 2747.54 & 189.380 & 0.033 & & \\
\hline 3 & & 14.438 & 2678.10 & 185.484 & 0.033 & & \\
\hline$\ldots$ & & & & & & & \\
\hline 30 & & 14.45 & 2608.01 & 180.535 & 0.033 & & \\
\hline
\end{tabular}

Table 2. b) vehicle B-The final calculation results of shortened time test. 


\begin{tabular}{|c|c|c|c|c|c|c|c|}
\hline \multirow[t]{2}{*}{ c } & $E_{R E E S S, C C P}$ & $d_{c}$ & $\Delta E_{R E E S S, \mathrm{c}}$ & $E C_{D C, c}$ & $K_{c}$ & $E C_{D C}$ & BER \\
\hline & Wh & $\mathrm{km}$ & Wh & $\mathrm{Wh} / \mathrm{km}$ & & $\mathrm{Wh} / \mathrm{km}$ & $\mathrm{km}$ \\
\hline 1 & \multirow{4}{*}{83971.131} & 14.491 & 2768.865 & 191.069 & 0.033 & \multirow{4}{*}{175.206} & \multirow{4}{*}{479.271} \\
\hline 2 & & 14.447 & 2633.326 & 182.278 & 0.031 & & \\
\hline 3 & & 14.409 & 2500.883 & 173.560 & 0.468 & & \\
\hline 4 & & 14.442 & 2531.102 & 175.260 & 0.468 & & \\
\hline
\end{tabular}

Surprisingly, the test on vehicle B showed the opposite result to vehicle A, that is, the BER in shortened time test is longer than in consecutive cycle test, approximately $+7 \%$ in value. The dataset $3^{\text {rd }}$ and $4^{\text {th }}$ with heavy weight $K_{c}$ in formula have low $E C_{D C, c}$, so the larger result of BER is calculated.

It is concluded that the results in Shortened time test would be deviated compared with consecutive cycle test, whereas the deviation value varies from vehicle to vehicle. The deviation of this two method of domestic vehicle A is small, while that of imported vehicle $\mathrm{B}$ is slightly larger.

\subsection{Results analysis}

Different vehicles perform individual control strategies under different battery state. As shown in Figure4, the braking energy regeneration system of vehicle $\mathrm{A}$ is forced to shut down when the battery is approximately full. It is obvious that the energy recovery almost zero in CLTC-P $1^{\text {st }}$ (label (1)). The phase Low of CLTC-P $2^{\text {nd }}$ (label (2)) has almost no energy recovery also. Not vehicle $\mathrm{B}$, just be weakened, however.

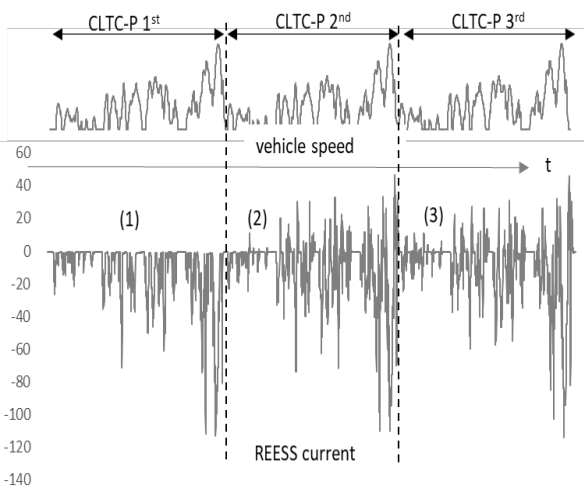

vehicle A

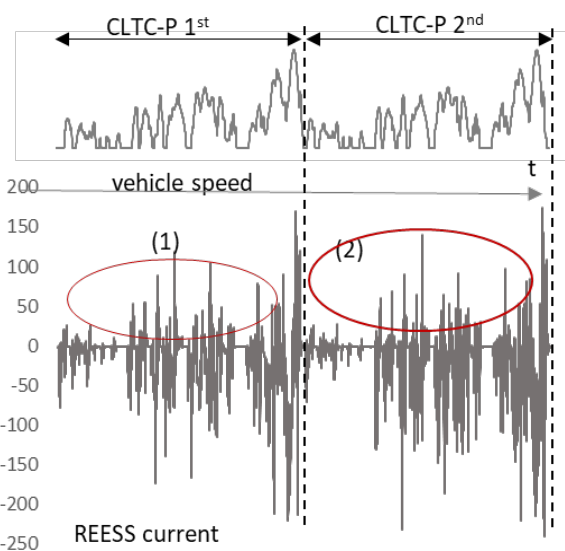

vehicle B

Fig. 4. Vehicle speed and REESS current of the first dataset in consecutive cycle test.

Energy control strategies, which varies between vehicles, would directly affect results of electric consumption. Figure 5 presents the electric consumption of dataset $\mathrm{c}^{\text {th }}\left(E C_{D C, c}\right)$ of vehicle $A$ and $B$.

In Figure 5(a), the value of dataset $1^{\text {st }} 160.57 \mathrm{kWh} / 100 \mathrm{~km}$ is much higher than others, approximately $25 \%$. From the dataset $2^{\text {nd }}$, the value gradually decreases, and after the dataset $4^{\text {th }}$, the value fluctuates little. The dotted line is the average value $125 \mathrm{kWh} / 100 \mathrm{~km}$ except dataset $1^{\text {st }}$ and $2^{\text {nd }}$. However, vehicle B is not quite the same, just as 5(c) shown. The first 13 values show a trend of gradual decrease. The latter values fluctuate slightly around $189 \mathrm{kWh} / 100 \mathrm{~km}$. And the value difference between cycle $1^{\text {st }}$ and other cycle of vehicle B is less than that of vehicle A. 
More importantly, same tendency, that is high energy consumption when the battery is sufficient, are reflected faithfully in Shortened time test as same as in consecutive cycle test. Compare 5(b) to 5(a), it is obvious that $1^{\text {st }}$ and $2^{\text {nd }}$ in 5(b) is near to the value in 5(a), similarly, the value of $3^{\text {rd }}$ and $4^{\text {th }}$ in $5(\mathrm{~b})$ is near to the average value in 5(a) as well.

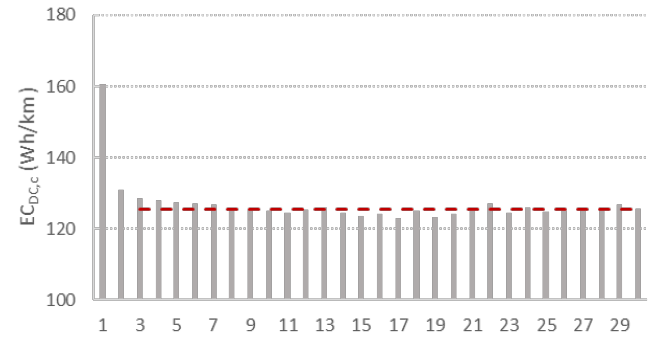

(a) consecutive cycle test of vehicle A

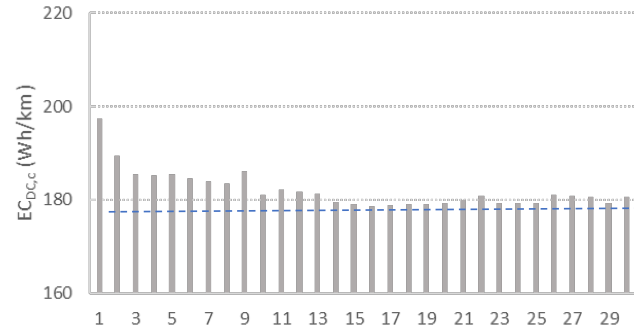

(c) consecutive cycle test of vehicle B

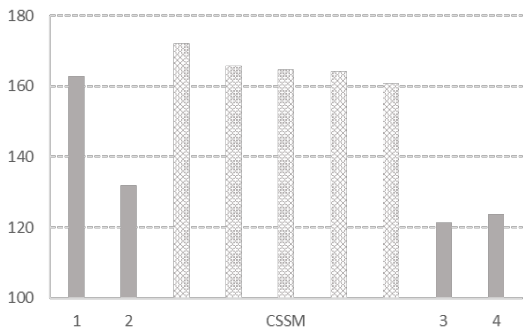

(b) shortened time test of vehicle A

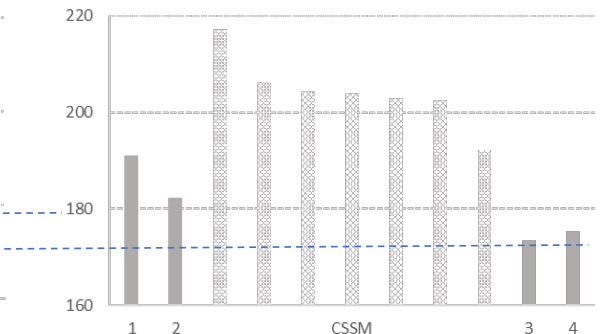

(d) shortened time test of vehicle B

Fig. $5 E C_{D C, C}$ in consecutive cycle test and Shortened time test ${ }^{1}$ of vehicle $\mathrm{A}$ and $\mathrm{B}$.

Note: ${ }^{1}$ CSSM is constant speed segment $(100 \mathrm{~km} / \mathrm{h})$. Divide these data into segments every 1800 second and calculation $E C_{D C, C}$, like the column filled with shadows.

In Figure 5(d), the value of dataset $3^{\text {rd }}$ is the minimum. And it is lower than all values in 5(c). Considering that the DS2 following the high-speed constant cycle, the vehicle is in good condition. For example, there is no frequent adjustment of motor controller because of the stable temperature state. And cause of constant speed, there is no frequent change of braking energy recovery. These above are likely to affect the energy consumption of accessories in the DS2. The resistance of lubrication system may be reduced also [11]. These are what we called "high-speed sequelae". The short-term impact may be small, but the cumulative results should not be ignored. The effect would not last long, because the high-speed sequelae have disappeared gradually with the continuous speed change under transient condition. It is obvious from 5(b) and 5(d) that the value of dataset $3^{\text {rd }}$ is less than the dataset $4^{\text {th }}$.

\subsection{Conclusions}

The Shortened time test time can be greatly reduced, approximately 60 percent, especially for vehicles with large battery capacity. In addition, the weighting factor setting of DS1 and DS2 is reasonable. It can be considered separately that effect of braking energy regeneration system under special conditions.

However, there are still some deviations between the Shortened time test results and consecutive test results by the same vehicle. The magnitude of the deviation is strongly related to the energy control strategy of vehicle. In this study, -2.3 percent and +7 percent are the BER deviation of vehicle A and B calculated by the two tests respectively. 
In order to avoid the "high speed sequelae", it is recommended that the vehicle be forced to break 10 minutes after the end of CSSM. The result would be closer to that of consecutive cycle test. Some comparative tests will be carried out in the future.

\section{Summary}

This paper is a research on the applicability of Shortened time method to test battery electric range of pure electric vehicles based on CLTC-P according to GB/T 18386.1-2020.

In this study, revisions of CLTC-P and Shortened time test and the results calculation were interpreted at first in the way of flowchart with annotation. To compare difference between Shortened time test and consecutive cycle test, two sets of experiments is carried out with an import car and a domestic car. The following conclusion are drawn from the comparative experiments.

(1) The Shortened time method can greatly reduce the test time and save a lot of equipment and human resources.

(2) There are still some deviations between the Shortened time test results and consecutive test results by the same vehicle.

(3) A method of decrease distinction is presented, that is the vehicle should be forced to break 10 minutes after the end of CSSM.

This study is supported by the National Key R\&D Program of China (2018YFB0106404) and Research and Development of Chinese New Energy Automobile Products Test Driving Cycles (CATC).

\section{Reference}

1. Jun Yong. Interpretation of the new energy vehicle subsidies in 2019[J]. Light Vehicles, 2019(Z2):37-39.

2. Ministry of Finance of the People's Republic of China. Circular on further improving the promotion and application of financial subsidy policies for new energy vehicles[Z]. 2019-3-26.

3. Shatrughan Modi, Jhilik Bhattacharya, Prasenjit Basak. Estimation of energy consumption of electric vehicles using Deep Convolutional Neural Network to reduce driver's range anxiety[J]. ISA Transactions,2020,98.

4. Xu Hao,Hewu Wang,Zhenhong Lin,Minggao Ouyang. Seasonal effects on electric vehicle energy consumption and driving range: A case study on personal, taxi, and ridesharing vehicles[J]. Journal of Cleaner Production,2020,249.

5. An Xiaopan. Analysis of travel characteristics and energy consumption of electric vehicle[D]. Hebei University of Technology, 2017.

6. Ministry of Industry and Information Technology of the People's Republic of China. The Parallel Corporate Average Fuel Consumption (CAFC) and New Energy Vehicle (NEV) Credit Schemes[Z]. 2017-09-28.

7. GB/T 18386-2020. Test Method for Energy Consumption and Range of Electric Vehicles- part1: Light-duty Vehicles[S]. 2020.

8. ECE/TRANS/WP.29/GRPE. Global technical regulation on Worldwide harmonized Light vehicles Test Procedures (WLTP). 2019.

9. GB/T 38146.1 China Automotive Test Cycle - Part 1: Light-duty vehicles[S].2019.

10. The Results of the Second Batch of EV-TEST in 2019 were Successfully Released in Tianjin [DB/OL]. http://ev-test.catarctc.com/. 2019-12-31. 
role does

car

lubricant

have[DB/OL]. http://mini.eastday.com/bdmip/180427104002639.html.

12. Guo yan, Liu ruifeng, Wang Qi. Comparison and Analysis of Pure Electric Vehicles Energy Consumption Standards GB/T 18386.1-XXXX and GB/T 18386-2017 [J]. China Auto, 2019(11):41-45. 